\title{
Cardiorenal Syndrome in Acute Heart Failure Syndromes
}

\author{
Mohammad Sarraf ${ }^{1}$ and Robert W. Schrier ${ }^{2}$ \\ ${ }^{1}$ University of Minnesota, USA \\ ${ }^{2}$ University of Colorado, USA \\ Correspondence should be addressed to Robert W. Schrier, robert.schrier@ucdenver.edu
}

Received 22 November 2010; Accepted 9 January 2011

Academic Editor: Alejandro Martín-Malo

Copyright (C) 2011 M. Sarraf and R. W. Schrier. This is an open access article distributed under the Creative Commons Attribution License, which permits unrestricted use, distribution, and reproduction in any medium, provided the original work is properly cited.

Impaired cardiac function leads to activation of the neurohumoral axis, sodium and water retention, congestion and ultimately impaired kidney function. This sequence of events has been termed the Cardiorenal Syndrome. This is different from the increase in cardiovascular complications which occur with primary kidney disease, that is, the so-called Renocardiac Syndrome. The present review discusses the pathogenesis of the Cardiorenal Syndrome followed by the benefits and potential deleterious effects of pharmacological agents that have been used in this setting. The agents discussed are diuretics, aquaretics, natriuretic peptides, vasodilators, inotropes and adenosine $\alpha 1$ receptor antagonists. The potential role of ultrafiltration is also briefly discussed.

\section{Introduction}

Patients with acute heart failure syndromes (AHFS) are usually admitted because of severe systemic congestion that frequently presents with dyspnea. Known as the hallmark of AHFS, congestion is mainly due to pulmonary venous hypertension (World Health Organization type 2). These patients may also present with low cardiac output and/or systemic hypotension. This has ranged from $<2 \%$ [1] to $7.7 \%$ [2] to $29 \%$ [3] depending on the series. Dyspnea is the most common symptom in these patients that implies an elevated pulmonary venous pressure that is often accompanied by increased central venous pressure (CVP) and/or peripheral edema. Therefore, the most reasonable therapeutic target is systemic congestion. There is substantial evidence that the main driver of morbidity, mortality, and readmission to the hospital is volume overload [1-5]. Moreover, it is well established that patients who are admitted with AHFS and renal dysfunction have worse outcomes $[1,5-9]$. In this paper, we discuss the pathophysiology of AHFS and its contribution to impairment of kidney function. In the end, we approach the current evidence of therapeutic strategies in patients with cardiorenal syndrome in AHFS.

\section{Pathophysiology}

As noted above, the hallmark of AHFS is congestion. The interaction between the heart and the kidney is modulated by the cardiorenal axis. The sympathetic nervous system (SNS), renin-angiotensin-aldosterone system (RAAS), and arginine vasopressin (AVP) are the primary neurohormones that maintain the integrity of effective arterial blood volume, hence the cardiorenal axis [4, 10-14].

In a nonfailing heart, an increase in left atrial pressure stimulates a feedback system, which decreases the release of AVP from posterior pituitary. This reflex is abolished by vagotomy. Furthermore, the elevated atrial pressure decreases the renal SNS stimulation. On the other hand, natriuretic peptides are released due to myocardial stretch and dilatation. The interaction of these pathways ultimately increases sodium and water excretion that maintains a steady state for total blood volume and preserves the integrity of the arterial circulation $[4,13]$ (Figure 1).

When heart failure develops, this physiologic response is disrupted and the kidneys continue to retain sodium and water despite an elevated total blood volume. However, the primary regulation of body fluid homeostasis is modulated 


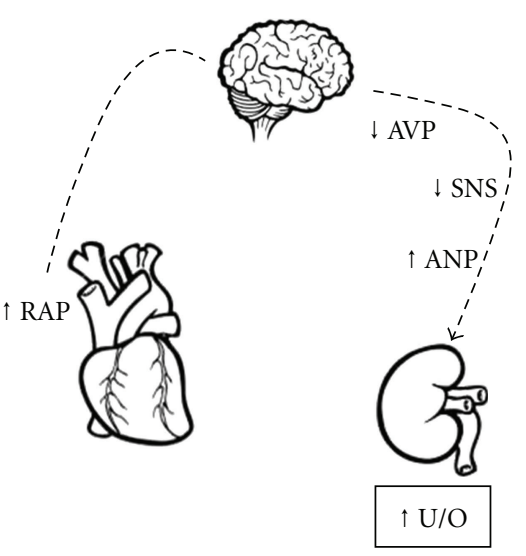

Figure 1: Physiologic interaction of neurohormones and maintenance of total blood volume. U/O: Urine output, $\uparrow$ : increased, RAP: right atrial pressure, ANP: atrial natriuretic peptide, SNS: sympathetic nervous system and AVP: arginine vasopressin.

by the smaller arterial circulation, enabling the system responsible for the perfusion of the body's vital organs to respond to small changes in body fluid volume $[4,10,11]$. This portion of blood volume only comprises $15 \%$ of the total blood volume $[10,11]$. Therefore, in a failing heart, despite elevated total sodium and total water and significant engorgement of the venous system, kidneys continue to retain sodium and water due to disrupted maintenance of body fluid homeostasis (Figure 2). This applies to patients with low or high cardiac output [10].

Elevated level of renin secretion is demonstrated in early stages of heart failure [14]. Renin stimulates angiotensin II (Ang-II) generation. Angiotensin II causes arterial vasoconstriction and therefore, increases cardiac afterload with a resultant decrease in stroke volume. Moreover, Ang-II stimulates the release of aldosterone and SNS. The activation of RAAS and SNS results in further vasoconstriction of the afferent arterioles of the kidneys $[4,13]$ that decreases renal blood flow and glomerular filtration rate (GFR). The stimulation of RAAS and SNS also increases proximal tubular absorption of sodium and decreases sodium delivery to the distal tubules and collecting ducts, which is the site of action of aldosterone [15]. Therefore, in patients with heart failure escape from the sodium-retaining effect of aldosterone on the distal nephrons, a phenomenon that normally occurs, is impaired. The end-result of this combined neurohormonal activation is continuous reabsorption of sodium and water which leads to congestion.

SNS is activated through increased Ang-II (see above) as well as activation of the baroreceptors in the aorta and aortic arch [11]. SNS has a positive feedback on RAAS stimulation that results in further stimulation of aldosterone. Aldosterone and Ang-II both accelerate fibrosis of the myocardium and remodeling of the failing heart [16]. Furthermore, neurohormones are strong mediators of oxidative injury, inflammation, and cell death that leads to widespread endothelial dysfunction. Thus, Ang-II exerts many deleterious effects through the activation of NADPH- and NADHoxidase. These enzymes are activated within vascular smooth muscle cells, cardiac myocytes, and renal tubular epithelial cells, generating superoxide, a reactive oxygen species with unfavorable effects [17]. More importantly, nitric oxide release by endothelium may be disturbed in the presence of superoxide and reactive oxygen species. This results in endothelial dysfunction, hypertension, and increased cardiac afterload [17]. As many as $50 \%$ of patients with AHFS present with a systolic blood pressure $>140 \mathrm{~mm} / \mathrm{Hg}$ [1]. Elevated blood pressure is present in patients either with systolic heart failure or heart failure with preserved ejection fraction [1].

AVP is a hormone that is secreted from the posterior pituitary and is normally suppressed by hypoosmolality. In the failing heart, however, even in the presence of hyponatremia, and thus hypoosmolality, there is a marked increase in AVP secretion secondary to nonosmotic baroreceptormediated release of the hormone. This phenomenon commonly overrides the osmotic release of AVP [18-20]. AVP activates the V2 receptor on the basolateral surface of principal cell of the collecting duct. This increases expression and trafficking of the aquaporin 2 water channel to the apical surface. The resultant increased water permeability of the collecting duct allows osmotic water equilibrium with the hypertonic interstitium and urinary concentration. Also, AVP stimulates the V1a receptors of the vascular smooth muscle that results in vasoconstriction of the arterial and venous system. Therefore, AVP increases preload and afterload through V2 and V1a receptor activation [4]. Thus, AVP potentially may result in further remodeling of the myocardium by these pathways (Figure 3 ).

\section{Goal of Therapy}

It is evident that the neurohormonal storm and worsening kidney function in AHFS ultimately ensue to venous congestion and elevated central venous pressure (CVP), which results in a vicious cycle. In a prospective cohort of 145 patients from the Cleveland Clinic, CVP was the most important hemodynamic factor causing worsening renal function in patients with AHFS [21]. In addition, in a retrospective analysis of 2557 patients who underwent cardiac catheterization for hemodynamic assessment, elevated CVP remained as the single most important prognostic factor for worsening renal function and mortality [22]. There is growing evidence that hypervolemia, that is, increased pulmonary capillary wedge pressure, independently correlates with mortality [23] and may predict an urgent need for cardiac transplantation [24]. Furthermore, improvement in cardiac output/index in patients with AHFS has little impact on outcome of patients with AHFS [25], even when therapy for decongestion is modulated carefully by invasive hemodynamic assessment [26]. Furthermore, transmission of venous pressure to renal venous system impairs renal blood flow and GFR. In 1861, Ludwig observed that as CVP increased above $10 \mathrm{~mm} / \mathrm{Hg}$, the urine output started to fall [27]. In 1931, Winton showed in isolated kidneys from dogs, that elevated venous pressure drops the urine output, while the arterial side was connected to a perfusing 


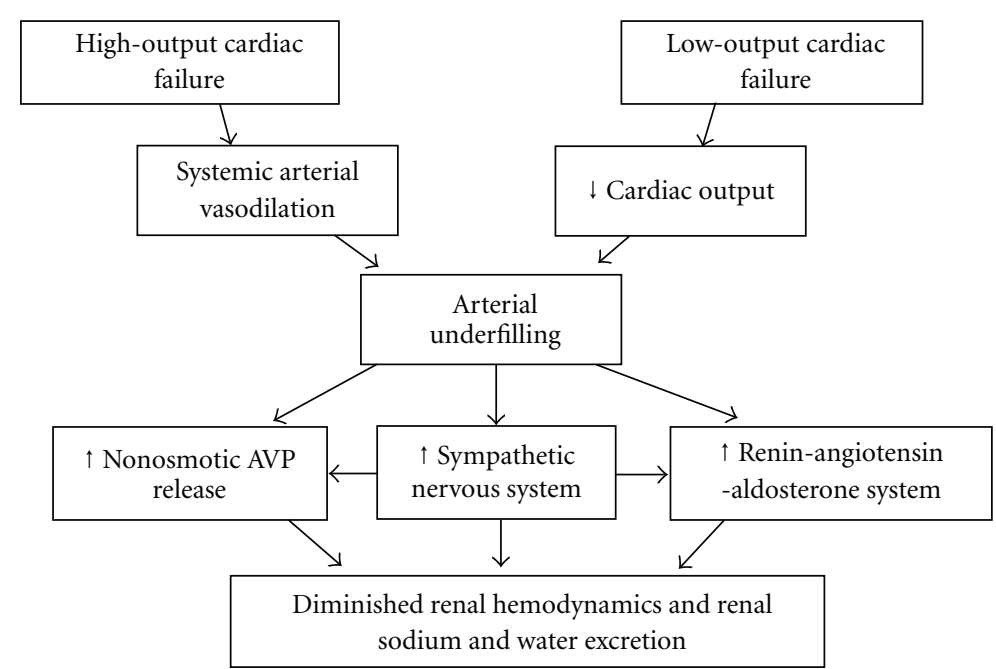

Figure 2: Pathophysiology of acute decompensated heart failure. (Reproduced with permission from [8].)

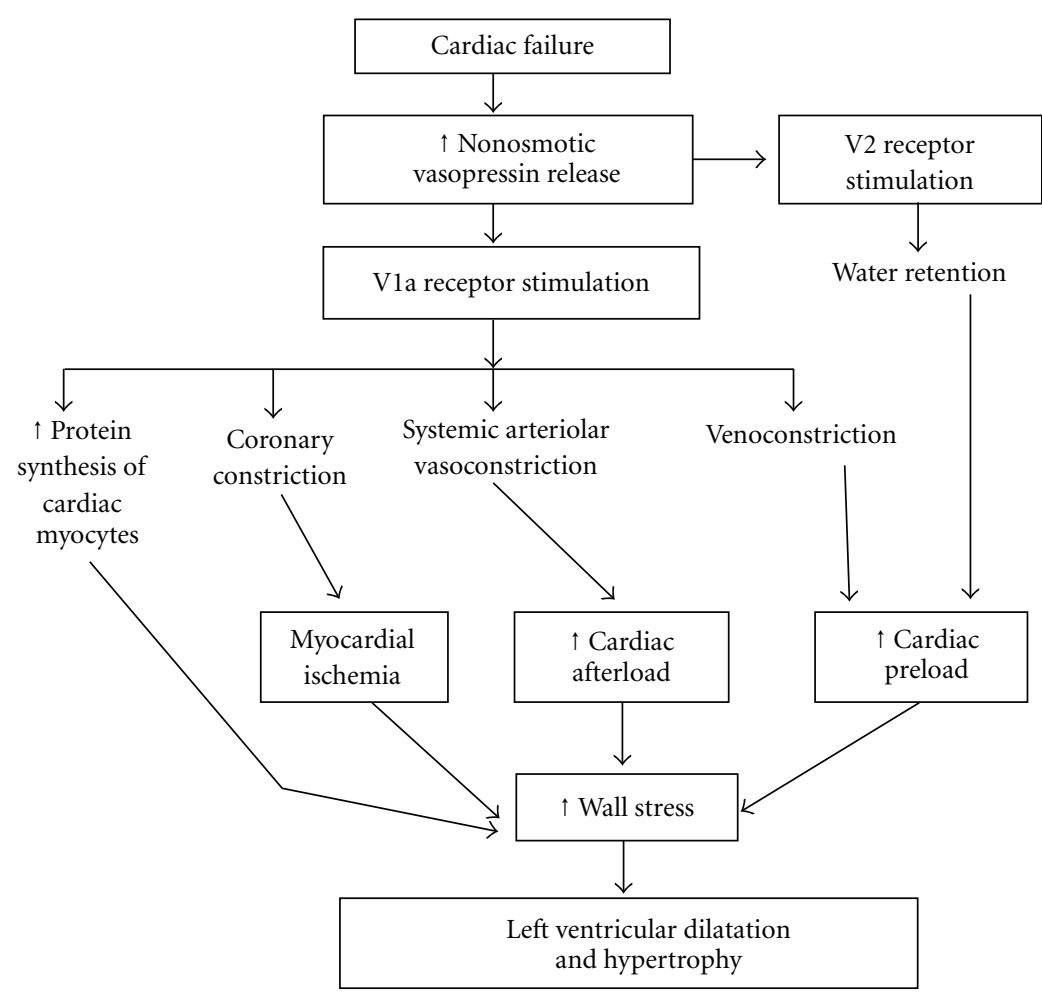

FIGURE 3: Vasopressin stimulation of V2 and V1a receptors can contribute to events that worsen cardiac function (with permission from [2].)

pump [28]. More recently, Mullens et al. demonstrated that intra-abdominal pressure (IAP) correlated with worsening renal function and lowering IAP improves renal function [29]. Thus, the focus of the clinician should be on reducing the congestion with as little hemodynamic compromise as possible. The rate of fluid removal, therefore, should not exceed the interstitial fluid mobilization rate (estimated at 12 to $15 \mathrm{~mL} / \mathrm{min}$ ), since it may further activate the RAAS and worsen the neurohormonal storm $[25,30]$.

\section{Treatment of Patients with AHFS}

Much of the challenge of the management of patients with cardiorenal syndrome who present with AHFS lies in the balance of decongestion and hemodynamic compromise. Ideally, one wishes to lower the preload, afterload and pulmonary capillary wedge pressure without reducing the blood pressure and GFR. Thus, the ideal agent should reduce left ventricular filling pressure, pulmonary 
TABLE 1: Pharmacologic agents in the management of patients with AHFS.

\begin{tabular}{|c|c|c|c|}
\hline Medication & Initial dose & Dose range & comments \\
\hline \multicolumn{4}{|c|}{ Diuretics } \\
\hline Furosemide & 20-80 mg IV bolus & $\begin{array}{l}20-400 \mathrm{mg} \text { boluses may } \\
\text { repeat q6-8 H }\end{array}$ & $\begin{array}{l}\text { Infusion is recommended at } 5 \text { to } 40 \mathrm{mg} / \mathrm{hr} \text {. If }>240 \mathrm{mg} / \mathrm{hr} \text {, } \\
\text { risk of ototoxicity increases }\end{array}$ \\
\hline Torsemide & $10-40 \mathrm{mg}$ bolus & 20-200 mg bolus & Continuous infusion: $5-20 \mathrm{mg} / \mathrm{hr}$ \\
\hline Bumetanide & $0.5-2 \mathrm{mg}$ bolus & $0.5-4 \mathrm{mg}$ bolus & Continuous infusion: $0.1-0.5 \mathrm{mg} / \mathrm{hr}$ \\
\hline \multicolumn{4}{|c|}{ Vasodilators } \\
\hline Nitroprusside & $0.3-0.5 \mu \mathrm{g} / \mathrm{kg} / \mathrm{min}$ & $0.3-5 \mu \mathrm{g} / \mathrm{kg} / \mathrm{min}$ & $\begin{array}{l}\text { Infusion rates of }>10 \mu \mathrm{g} / \mathrm{kg} / \mathrm{min} \text { may cause cyanide } \\
\text { toxicity. Also, caution during active myocardial ischemia }\end{array}$ \\
\hline Nitroglycerine & $10-20 \mu \mathrm{g} / \mathrm{min}$ & $20-400 \mu \mathrm{g} / \mathrm{min}$ & severe headache, hypotension, closed-angle glaucoma \\
\hline Nesiritide & NO BOLUS & $0.005-0.03 \mu \mathrm{g} / \mathrm{kg} / \mathrm{min}$ & $\begin{array}{c}\text { Titration: increase infusion rate by } 0.005 \mu \mathrm{g} / \mathrm{kg} / \mathrm{min} \text { (no } \\
\text { more than every } 3 \mathrm{hr} \text {, up to a maximum of } \\
0.03 \mu \mathrm{g} / \mathrm{kg} / \mathrm{min} \text { ) }\end{array}$ \\
\hline \multicolumn{4}{|c|}{ Inotropes } \\
\hline Dopamine & $2-5 \mu \mathrm{g} / \mathrm{kg} / \mathrm{min}$ & $2-20 \mu \mathrm{g} / \mathrm{kg} / \mathrm{min}$ & May increase mortality. Caution for arrhythmia \\
\hline Dobutamine & $1-2 \mu \mathrm{g} / \mathrm{kg} / \mathrm{min}$ & $1-20 \mu \mathrm{g} / \mathrm{kg} / \mathrm{min}$ & May increase mortality. Caution for arrhythmia \\
\hline Milrinone & $\begin{array}{l}50 \mu \mathrm{g} / \mathrm{kg} \text { IV loading dose } \\
\text { over } 10 \mathrm{~min} \text {; then } \\
0.25-1.0 \mu \mathrm{g} / \mathrm{kg} / \mathrm{min} \text { infusion }\end{array}$ & $0.10-0.75 \mu \mathrm{g} / \mathrm{kg} / \mathrm{min}$ & May increase mortality. Caution for arrhythmia \\
\hline \multicolumn{4}{|c|}{ Other } \\
\hline Levosimendan & $\begin{array}{l}0.05-0.2 \mu \mathrm{g} / \mathrm{kg} / \mathrm{min} \text { bolus } \\
\text { over } 10 \mathrm{~min} \text { followed by } \\
\text { infusion }\end{array}$ & $0.5-2.0 \mu \mathrm{g} / \mathrm{kg} / \mathrm{min}$ & $\begin{array}{l}\text { May increase mortality. Not approved in the US. Caution } \\
\text { for hepatic impairment and LV outflow obstruction }\end{array}$ \\
\hline
\end{tabular}

congestion, improve renal function, preserve myocardial tissue, reduce neurohormonal levels, and not increase the incidence of arrhythmias. Unfortunately, to date there is no such medical regimen available. Since congestion is the hallmark of heart failure and correlates with morbidity and mortality in short, and long-term studies (see above), the primary aim of therapy should focus on decongestion with the goals described above.

\section{Pharmacologic Approach}

We divide therapy to pharmacologic and nonpharmacologic interventions with the latest available evidence. For pharmacologic approach, one may utilize diuretics, aquaretics (V2 receptor antagonists), vasodilators, and inotropes. For nonpharmacologic approach, there is a paucity of welldone randomized control data in patients with AHFS; nevertheless, we will discuss the role of ultrafiltration in patients with AHFS.

5.1. Diuretics. Heart Failure Society of America guidelines recommends loop diuretics as the corner stone of therapy in patients with congestive symptoms in the setting of AHFS [31]. More than $88 \%$ of patients receive loop diuretics, mainly intravenously [1]. There are no randomized controlled trials to have evaluated the outcome studies of loop diuretics, but it is evident that patients with AHFS should not be left in the congestive state. As stated earlier, congestion correlates with mortality. Loop diuretics relieve the symptoms, even before diuresis [31] and also reduce the wedge pressure and left ventricular filling pressure. In patients who have severe congestion and renal dysfunction, diuresis may improve kidney function, possibly through relieving the venous and/or abdominal congestion. The dilemma, however, is the fact that there is no prognostication as to which patient will improve or worsen with diuretic therapy. In Table 1 are listed the diuretics in clinical practice for AHFS.

Comprehensive HFSA guideline recommends switching from intravenous to continuous infusion in patients who seem to be nonresponsive to diuretics [31]. This question has been recently investigated in a randomized double-blind, double dummy controlled trial in a $2 \times 2$ factorial design in Diuretic Optimization Strategies Evaluation (DOSE) trial [30]. In this study, the investigator concluded there was no statistically significant difference in global symptom relief or change in renal function at 72 hours (coprimary endpoint) between intermittent versus continuous infusion or low dose ( $\times 1$ of the oral outpatient dose) versus high dose $(\times 2.5$ times of the oral outpatient dose) [32]. This is the first randomized, controlled exploration of a management strategy in loop diuretics in AHFS patients. However, the high-dose strategy caused a mild renal dysfunction that was reversible within 


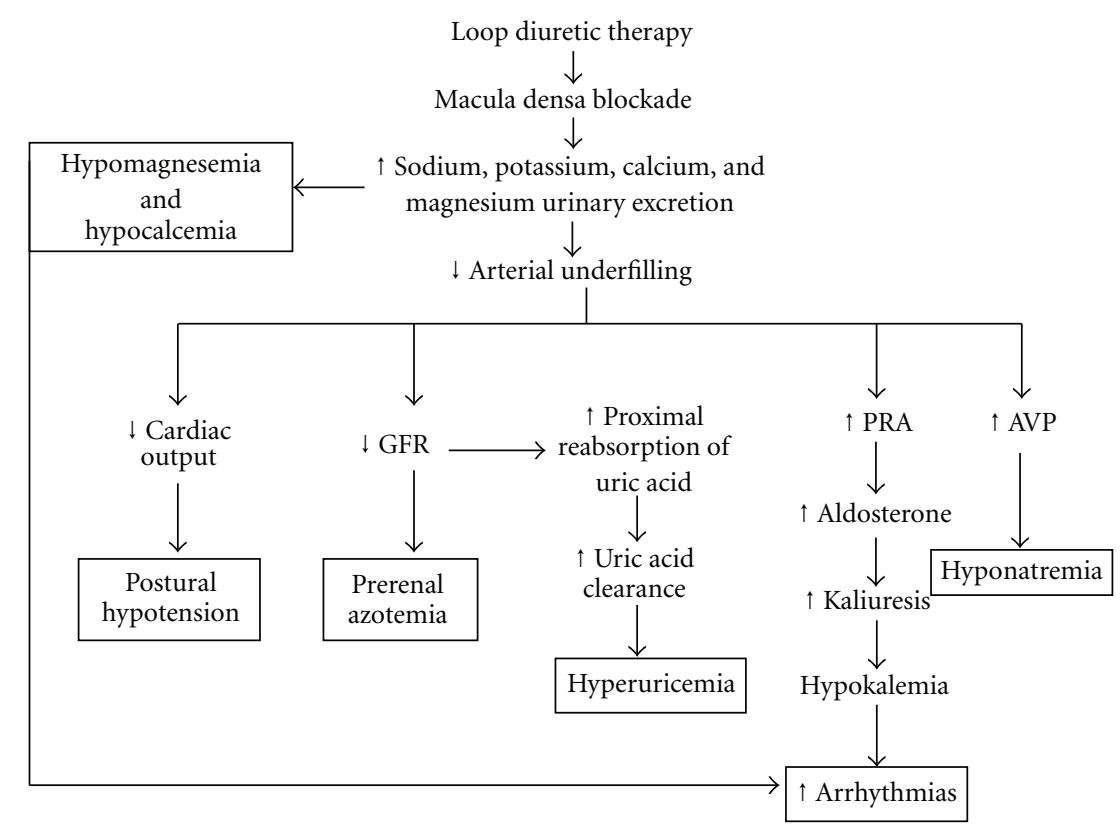

Figure 4: The mechanisms of adverse effects of loop diuretics (with permission from [11]).

one week. There was also no evidence for increased risk of clinical events at 60 days after high-dose therapy or after lowdose, continuous, or intermittent diuretic therapy. On the other hand, patients in the high-dose group, compared with the low-dose group, showed significant improvements in a series of secondary end points assessed at 72 hours, including weight loss, heart-failure biomarkers and dyspnea [32].

Despite all the benefits of loop diuretics in acute setting there are serious adverse effects associated with these agents [13]. Loop diuretics frequently develop electrolyte abnormalities, mainly hyponatremia, hypokalemia and hypomagnesemia. The loop diuretics inhibit the macula densa of the nephron. This results in further release of renin and stimulation of neurohormones and acute vasoconstriction response after administering loop diuretics (Figure 4) [33]. The vasoconstriction can reduce the GFR by further afferent vasoconstriction, which may occur despite substantial increase in urine output [34]. However, improved myocardial function may develop due to reduction in ventricular size and wall stress. This may ultimately diminish mitral regurgitation and improve cardiac output and GFR [13]. Hypotension does not frequently happen when using diuretics, but it may occur in the presence of generous doses of vasodilators.

Another important dilemma in managing these patients is diuretic resistance. Patients with AHFS have significant neurohormonal activation and may have chronic kidney dysfunction [1]. Using loop diuretics may quickly worsen the compromised GFR and further enhance neurohormonal stimulation. In addition, there is a potential hypertrophy of the distal tubule in these patients that further limits the kidney's response to diuretics $[35,36]$. To overcome diuretic resistance, it is always a reasonable approach to limit total daily sodium intake to less than 2 gm. HFSA guideline recommends fluid restriction of 2 liters and if patient has moderate hyponatremia $(<130 \mathrm{mEq} / \mathrm{L})$, more aggressive fluid restriction [31]. The pharmacologic approach to overcome resistance is to add another diuretic that blocks the distal tubule, such as a thiazide diuretic. Another approach is adding metolazone but consideration of the agent's long half life is very important ( $\sim$ days). Lastly, switching from intermittent to continuous infusion may be considered, although as stated above, in the randomized control study, the outcome was not different. At the time of writing of this paper further details of the DOSE trial are not available.

5.1.1. Mineralocorticoid Antagonists. About 50\% of patients with AHFS have heart failure with preserved ejection fraction (HFPEF) [1]. In these patients, the neurohormonal stimulation is independent of the cardiac output or ejection fraction of the patient. On the other hand, cirrhotic patients share a common pathophysiology for sodium and water retention by neurohormonal activation secondary to splanchnic vasodilation. As stated earlier, patients with heart failure cannot escape from the sodium-retaining effect of aldosterone. The same is true for cirrhotic patients with ascites. Thus, patients with heart failure have a similar pathophysiology of cirrhotic patients. Both patient populations have secondary hyperaldosteronism. The diuretic of choice in cirrhosis is mineralocorticoid antagonists, not a loop diuretic [37]. They are used as the mainstay of therapy since they target a primary underlying pathophysiology of the disease namely, secondary hyperaldosteronism. Loop diuretics are utilized in cirrhosis as an adjunct. In the Randomized Aldactone Evaluation Study (RALES), spironolactone was used as $25 \mathrm{mg}$ orally once a day [38]. It was shown that this dose did not decrease sodium retention. The interpretation of the RALES 
study therefore was that the results were due to nongenomic, nonnatriuretic effects of spironolactone on myocardium by preventing further remodeling and/or reducing fibrosis [38]. There are limited data for using the natriuretic doses of spironolactone (at least $50 \mathrm{mg}$ orally once a day) in patients with heart failure. To our knowledge, other than a small report, there are no randomized well-conducted studies available to evaluate the effect of natriuretic doses of spironolactone in heart failure patients. In 1965, Braunwald et al. conducted a small trial of spironolactone in 3 patients with heart failure (mainly due to valvular disease). The dose of spironolactone utilized in that study was $100 \mathrm{mg}$ orally once a day and sodium excretion increased [39]. In a small prospective study in patients with severe heart failure and confirmed diuretic resistance, the effect of spironolactone was investigated [38]. All medications were discontinued. Spironolactone was started at $200 \mathrm{mg}$ orally twice a day, while the patients were taken off supplemental potassium. Over a 4-day period, spironolactone completely abolished the urinary sodium retention and atrial natriuretic peptide diminished substantially [40].

Since the publication of RALES study, there has been some concern regarding the risk of hyperkalemia. This cautionary note is mainly based on one epidemiologic study from Canada. In this study, the investigators observed a statistically significant increased risk of hospitalization and hospital mortality in association with hyperkalemia after publication of the RALES trial [41]. However, a subanalysis of Eplerenone Post-Acute Myocardial Infarction Heart Failure Efficacy and Survival Study (EPHESUS) showed that eplerenone (dose range of 25 to $50 \mathrm{mg} / \mathrm{d}$ ) in postmyocardial infarction patients with heart failure and/or LV ejection fraction (LVEF $<40 \%$ ) did not significantly increase the risk of hyperkalemia [42]. Furthermore, in another study from Scotland, in a population-based longitudinal analysis in patients with or without heart failure, there has been no increase in hospitalizations for hyperkalemia between 1994 to 2007 [43]. Nevertheless, with the paucity of results with natriuretic doses of mineralocorticoid antagonists ( $>25 \mathrm{mg} /$ day of spironolactone or $>50 \mathrm{mg}$ of eplerenone), prospective, randomized studies need to be performed in patients with AHFS, in the presence of a low-potassium diet and a potassium-losing loop diuretic, to block the sodium retaining effect of aldosterone by careful titrating of spironolactone doses greater than $25 \mathrm{mg} / \mathrm{d}$. If shown to be effective in treating congestion in AHFS, this could alter the frequent rehospitalization for congestion and the discharge of AHFS patients with continued symptoms of congestion (estimated to be $\sim 50 \%$ ).

5.2. Vasopressin Antagonists (Aquaretics). The only pharmacologic agent other than loop diuretics that is capable of rapid diuresis in AHFS is vasopressin antagonist. V2 receptors are stimulated by AVP and increase the aquaporins (see above) on the distal nephrons and increases permeability to water. A profound water diuresis (aquaresis) occurs by blocking the V2 receptors. Unlike any other diuretics, V2 antagonists do not affect the urinary excretion of electrolytes. In fact, in patients with hyponatremia, the serum sodium concentration normalizes while the intravascular volume is decreasing. Currently, there are 2 vasopressin antagonists available in the U.S., conivaptan and tolvaptan. Conivaptan is a mixed antagonist (V1a and V2 antagonist) and tolvaptan is a selective V2 receptor antagonist. The indication for use for both agents is the presence of hyponatremia and heart failure. It is not indicated for hypervolemia in the absence of hyponatremia [44].

In the Acute and Chronic Therapeutic Impact of a Vasopressin antagonist in Congestive Heart Failure (ACTIV) trial, 319 patients with heart failure were randomized in 3 different doses of tolvaptan [45]. Tolvaptan produced a significant decrease in body weight throughout hospitalization and a modest improvement in HF symptoms without any adverse hemodynamic compromise, electrolytes abnormalities, or renal dysfunction. A post-hoc analysis of this study demonstrated that mortality was reduced in patients with renal dysfunction or severe systemic congestion in the tolvaptan arm [44]. In a followup study of the Efficacy of Vasopressin Antagonism in Heart Failure Outcome Study With Tolvaptan (EVEREST) trial, patients were randomized to tolvaptan or placebo and followed for short term and long-term (median follow-up of 9.9 months) outcomes [46]. While tolvaptan produced a substantial normalization of the serum sodium concentration and significant weight loss in comparison to placebo, these findings did not translate to beneficial effect on readmission for heart failure or mortality [46]. EVEREST did show a statistically significant decrease in dyspnea during the first week on congestion. Thus far, there is only one study in AHFS patients with a mixed V1a/V2 antagonist (conivaptan) [47]. In this pilot study with conivaptan, there was a marked aquaresis in heart failure patients without any hemodynamic compromise [47]. Whether this agent improves outcomes of patients with AHFS remains to be elucidated.

5.3. Natriuretic Peptides. Brain natriuretic peptides (BNP) act upon guanylyl cyclase-linked natriuretic peptide receptors A and B. The downstream pathway of stimulation of these receptors is increased cyclic GMP production. Natriuretic peptides reduce the cardiac filling pressure and can improve symptoms of dyspnea as shown in Vasodilatation in the Management of Acute CHF study (VMAC) [48]. Nesiritide, a synthetic natriuretic peptide, was able to reduce significantly the pulmonary capillary wedge pressure in 15 minutes when compared to placebo, but not when compared to nitroglycerine [48]. Subsequently, significant concern was raised against nesiritide by a meta-analysis of randomized controlled trials that found a significant increased risk of worsening renal function [49]. In a retrospective study from the Mayo Clinic, Riter et al. demonstrated if these agents are judiciously used in low doses without an initial bolus (as was the case in the trials), the renal function improves $[13,50]$. This approach has not been studied in a randomized controlled trial.

5.4. Vasodilators. Decongestion is the center focus of treatment of patients with AHFS with the expectation that as intravascular volume falls, cardiac filling pressures will 
decline and symptoms improve. Therefore, it is a reasonable approach to target the systemic vascular resistance. By decreasing the vascular resistance, the cardiac filling pressures, pulmonary and systemic congestion may be alleviated and ventricular systolic and diastolic function may improve.

Nitroglycerine (NTG) improves the hemodynamics by decreasing right atrial pressure, pulmonary capillary wedge pressure, and reducing afterload [13]. These changes have a substantial effect on the congestive state of the patient. Furthermore, by reducing the preload, the myocardial stretch is diminished and the myocardial wall stress declines substantially. While all of these changes intuitively make therapeutic sense, there has never been any study in patients with AHFS randomized to NTG versus placebo. There is only one study of comparison of high-dose NTG and low-dose furosemide versus low-dose NTG and high-dose furosemide in patients with AHFS that was in favor of high-dose NTG and low-dose furosemide [51]. While NTG may decrease $\mathrm{BNP}$, there is a concern for renin elevation, most likely due to hypotension [52]. There is ample evidence that NTG increases coronary flow, but whether the coronary circulation changes in AHFS due to elevated LVEDP and reduced coronary perfusion pressure are beneficial is not well studied. This is particularly important in heart failure patients with ischemic cardiomyopathy. Judicious use of NTG in appropriately selected patients seems to be quite safe (Table 1).

Nitroprusside is a very potent balanced venous and arterial vasodilator. It remains the reference vasodilator for severe acute low-output left-sided heart failure as long as the arterial pressure is reasonable. In patients with congestion and acute myocardial infarction, nitroprusside is the agent of choice. In patients who present with acute or chronic heart failure, it is still a very reasonable option, as long as the clinician is aware of the potential side effects of nitroprusside. The most important side effects is the precipitous fall in blood pressure (that should be avoided in AHFS), possible coronary steal, and thiocyanate toxicity, which can be fatal if not treated promptly [15].

5.5. Inotropes. In extreme conditions when the cardiac output is compromised, there is a clear indication for using inotropes. Once very well received in intensive care units, inotropes now are utilized less often except for condition just described. There is substantial evidence in large randomized clinical trials as well as retrospective studies that these agents significantly increase mortality despite all the desirable effects on hemodynamics including increasing cardiac output and reducing systemic vascular resistance. With the exception of dopamine none of the inotropes have any effects on renal hemodynamics.

Dobutamine is a synthetic catecholamine that acts on $\beta 1$ and weakly on $\beta 2$ receptors. The $\beta 1$ receptor has a vasodilatory effect on the vascular smooth muscle and positive inotropy on the myocardium. So, by improving the contraction of myocardium and reducing afterload the cardiac output improves. Milrinone blocks the phosphodiesterase inhibitor III that ordinarily deactivates cyclic AMP (cAMP). The increased cytosolic level of cAMP improves the myocardial function and decreases the vascular tone similar to dobutamine, but by a different mechanism. A novel inotrope that is not approved by FDA in the US is levosimendan. This agent stabilizes the conformational change of troponin to calcium and increases contraction. There was a randomized study with and without levosimendan in patients with severe heart failure which demonstrated an improvement in renal function with levosimendan [53].

Dobutamine has improved the symptoms in heart failure up to 30 days $[54,55]$. In a large registry of patients with AHFS, however, dobutamine was associated with a marked increase in mortality when compared to NTG [56]. In the Intravenous Milrinone for Exacerbations of Chronic Heart Failure (OPTIME-CHF), 951 patients without cardiogenic shock were randomized to milrinone versus placebo. The main outcome of the study was the cumulative days of hospitalization for cardiovascular cause within 60 days following randomization. There was no statistically significant difference between the groups. There was a trend towards higher mortality in milrinone group $(P=.19)$. Patients in milrinone arm had more episodes of hypotension that required intervention compared to placebo [57]. In the Randomized Multicenter Evaluation of Intravenous Levosimendan Efficacy (REVIVE) II study, the investigator demonstrated a significant symptomatic improvement of $33 \%$ in levosimendan arm [58]. But, this finding was negated by a trend toward increased mortality in patients randomized to levosimendan [59].

The routine use of inotropes, therefore, is not recommended unless the patient's hemodynamics are severely compromised. It is evident that all inotropes may cause harm, mainly by increased mortality. In the era of beta blockade as one of the main treatments for chronic heart failure, many patients may present with AHFS while they are on beta blockers. There are no data as to whether it is safe or unsafe to stop the beta blockade, but a recommendation to decrease the dose by $50 \%$ and continue with inotrope of choice seems reasonable. It is important to note that among all inotropes, dobutamine and dopamine act upon beta receptors. So, if it is possible to use milrinone that has a different mechanism of action and there is no need to stop beta blockers unless the patient is in preshock or shock state that prompts the physician to stop the beta blockers immediately.

5.6. Adenosine $\alpha 1$ Receptor Antagonists. Adenosine is markedly elevated in patients with AHFS. Since adenosine has a profound vasoconstrictor effect on the glomeruli, it is theoretically attractive to hypothesize that blockade of adenosine would improve the kidney function and outcomes. Since adenosine acts upon $\alpha 1$ receptor on the afferent glomerulus, the agent of choice should be a selective $\alpha 1$ adenosine receptor blocker. In the Placebo-Controlled Randomized Study of the Selective A1 Adenosine Receptor Antagonist Rolofylline for Patients Hospitalized with Acute Decompensated Heart Failure and Volume Overload to Assess Treatment Effect on Congestion and Renal Function (PROTECT) a randomized, double-blind controlled study with rolofylline failed to show any difference as 
to the primary composite end point of persistent renal impairment, hospital readmission, or death in up to 60 days after admission [60].

5.7. Ultrafiltration. Theoretically it is very reasonable to approach to patients with AHFS who present with significant volume overload. Ultrafiltration bypasses the kidney and there is virtually no immediate neurohormonal stimulation as occurs with loop diuretics blocking the macula densa. In the Ultrafiltration versus Intravenous Diuretics for Patients Hospitalized for Acute Decompensated Heart Failure (UNLOAD) trial, there was a marked decrease in body weight, vasoactive drug requirement as well as hospital readmission in 90 days in the ultrafiltration arm [61]. However, this was associated with a trend towards higherserum creatinine level in the first week of therapy in the ultrafiltration arm. One critique to this study may be the fact that patients in the diuretic arm were not very aggressively diuresed. So, it may be more difficult to demonstrate such beneficial effects if compared with diuretic therapy of comparable negative fluid balance. Nonetheless, it is a reasonable option in patients who are left in congestive state and make little urine despite maximal medical therapy. There is a recent study comparing the effects of ultrafiltration versus diuretics in decompensated heart failure. Ultrafiltration showed a greater clinical and hemodynamic improvement, as well as a decrease in aldosterone and $\mathrm{N}$ - terminal pro-B-type natriuretic peptide [62].

\section{Conclusion}

Cardiorenal syndrome is frequently present in patients who present with AHFS. The main driver of the pathophysiology and symptomatology of the patients is congestion. The focus of treatment should be relieving the congestion without perturbing the hemodynamics of the cardiorenal axis. As discussed in this paper, unfortunately every modality of treatment has beneficial and detrimental effects on this axis. Loop diuretics relieve congestion but stimulate the neurohormones and reduce GFR. Inotropes improve hemodynamics but can potentially increase mortality and arrhythmias. Aquaretics have not been proven to decrease mortality in a large randomized control trial, although there are no large data on mixed receptor blockers. Natriuretic peptides may worsen the kidney function and possibly increase mortality. Vasodilators can cause substantial hypotension while improving the hemodynamics. There is very little data about the use of natriuretic doses of mineralocorticoid receptor antagonists in severe heart failure. For these reasons, it is not possible to give one set of hard and fast rules to treat the AHFS patients who present with cardiorenal syndrome. This is left to the astute clinician to take advantage of every agent at the appropriately selected patients.

\section{References}

[1] K. F. Adams Jr., G. C. Fonarow, C. L. Emerman et al., "Characteristics and outcomes of patients hospitalized for heart failure in the United States: rationale, design, and preliminary observations from the first 100,000 cases in the Acute Decompensated Heart Failure National Registry (ADHERE)," American Heart Journal, vol. 149, no. 2, pp. 209216, 2005.

[2] L. Tavazzi, A. P. Maggioni, D. Lucci et al., "Nationwide survey on acute heart failure in cardiology ward services in Italy," European Heart Journal, vol. 27, no. 10, pp. 1207-1215, 2006.

[3] F. Zannad, A. Mebazaa, Y. Juillière et al., "Clinical profile, contemporary management and one-year mortality in patients with severe acute heart failure syndromes: the EFICA study," European Journal of Heart Failure, vol. 8, no. 7, pp. 697-705, 2006.

[4] R. W. Schrier, "Role of diminished renal function in cardiovascular mortality: marker or pathogenetic factor?" Journal of the American College of Cardiology, vol. 47, no. 1, pp. 1-8, 2006.

[5] G. C. Fonarow, W. T. Abraham, N. M. Albert et al., "Influence of a performance-improvement initiative on quality of care for patients hospitalized with heart failure: results of the organized program to initiate lifesaving treatment in hospitalized patients with heart failure (OPTIMIZE-HF)," Archives of Internal Medicine, vol. 167, no. 14, pp. 1493-1502, 2007.

[6] S. S. Gottlieb, W. Abraham, J. Butler et al., "The prognostic importance of different definitions of worsening renal function in congestive heart failure," Journal of Cardiac Failure, vol. 8, no. 3, pp. 136-141, 2002.

[7] G. C. Fonarow, J. T. Heywood, P. A. Heidenreich, M. Lopatin, and C. W. Yancy, "Temporal trends in clinical characteristics, treatments, and outcomes for heart failure hospitalizations, 2002 to 2004: findings from Acute Decompensated Heart Failure National Registry (ADHERE)," American Heart Journal, vol. 153, no. 6, pp. 1021-1028, 2007.

[8] L. Klein, B. M. Massie, J. D. Leimberger et al., "Admission or changes in renal function during hospitalization for worsening heart failure predict postdischarge survival. Results from the Outcomes of a Prospective Trial of Intravenous Milrinonoe for exacerbations of Chronic Heart Failure (OPTIME-CHF)," Circulation: Heart Failure, vol. 1, pp. 25-33, 2008.

[9] R. W. Schrier, "Blood urea nitrogen and serum creatinine. Not married in heart failure," Circulation: Heart Failure, vol. 1, pp. $2-5,2008$.

[10] R. W. Schrier, "Body fluid volume regulation in health and disease: a unifying hypothesis," Annals of Internal Medicine, vol. 113, no. 2, pp. 155-159, 1990.

[11] R. W. Schrier, "Pathogenesis of sodium and water retention in high-output and low-output cardiac failure, nephrotic syndrome, cirrhosis, and pregnancy (1)," The New England Journal of Medicine, vol. 319, no. 16, pp. 1065-1072, 1988.

[12] R. W. Schrier and W. T. Abraham, "Hormones and hemodynamics in heart failure," The New England Journal of Medicine, vol. 341, no. 8, pp. 577-585, 1999.

[13] M. Sarraf, A. Masoumi, and R. W. Schrier, "Cardiorenal syndrome in acute decompensated heart failure," Clinical Journal of the American Society of Nephrology, vol. 4, no. 12, pp. 2013-2026, 2009.

[14] R. Blankstein and G. L. Bakris, "Renal hemodynamic changes in heart failure," Heart Failure Clinics, vol. 4, no. 4, pp. 411423, 2008.

[15] H. H. Chen and R. W. Schrier, "Pathophysiology of volume overload in acute heart failure syndromes," American Journal of Medicine, vol. 119, supplement 1, no. 12, pp. S11-S16, 2006.

[16] W. Weber, "Mechanisms of disease: aldosterone in chronic heart failure," The New England Journal of Medicine, vol. 345, pp. 1689-1697, 2001. 
[17] J. S. Bock and S. S. Gottlieb, "Cardiorenal syndrome: new perspectives," Circulation, vol. 121, no. 23, pp. 2592-2600, 2010.

[18] R. W. Schrier, T. Berl, and R. J. Anderson, "Osmotic and nonosmotic control of vasopressin release," American Journal of Physiology, vol. 236, no. 4, pp. F321-332, 1979.

[19] R. W. Schrier and T. Berl, "Nonosmolar factors affecting renal water excretion (first of two parts)," The New England Journal of Medicine, vol. 292, no. 2, pp. 81-88, 1975.

[20] R. W. Schrier and T. Berl, "Nonosmolar factors affecting renal water excretion (second of two parts)," The New England Journal of Medicine, vol. 292, no. 3, pp. 141-145, 1975.

[21] W. Mullens, Z. Abrahams, G. S. Francis et al., "Importance of venous congestion for worsening of renal function in advanced decompensated heart failure," Journal of the American College of Cardiology, vol. 53, no. 7, pp. 589-596, 2009.

[22] K. Damman, V. M. van Deursen, G. Navis, A. A. Voors, D. J. van Veldhuisen, and H. L. Hillege, "Increased central venous pressure is associated with impaired renal function and mortality in a broad spectrum of patients with cardiovascular disease," Journal of the American College of Cardiology, vol. 53, no. 7, pp. 582-588, 2009.

[23] M. H. Drazner, J. E. Rame, L. W. Stevenson, and D. L. Dries, "Prognostic importance of elevated jugular venous pressure and a third heart sound in patients with heart failure," The New England Journal of Medicine, vol. 345, no. 8, pp. 574-581, 2001.

[24] A. S. Androne, K. Hryniewicz, A. Hudaihed, D. Mancini, J. Lamanca, and S. D. Katz, "Relation of unrecognized hypervolemia in chronic heart failure to clinical status, hemodynamics, and patient outcomes," American Journal of Cardiology, vol. 93, no. 10, pp. 1254-1259, 2004.

[25] M. Jessup and M. R. Costanzo, "The cardiorenal syndrome: do we need a change of strategy or a change of tactics?" Journal of the American College of Cardiology, vol. 53, no. 7, pp. 597-599, 2009.

[26] A. Nohria, V. Hasselblad, A. Stebbins et al., "Cardiorenal interactions: insights from the ESCAPE trial," Journal of the American College of Cardiology, vol. 51, no. 13, pp. 1268-1274, 2008.

[27] C. Ludwig, Lehrbuch d. Physiol, 2nd edition, 1861.

[28] F. R. Winton, "The influence of venous pressure on the isolated mammalian kidney," The Journal of Physiology, vol. 72, no. 1, pp. 49-61, 1931.

[29] W. Mullens, Z. Abrahams, H. N. Skouri et al., "Elevated intraabdominal pressure in acute decompensated heart failure. A potential contributor to worsening renal function?" Journal of the American College of Cardiology, vol. 51, no. 3, pp. 300-306, 2008.

[30] P. Fauchauld, "Effects of ultrafiltration of body fluid and transcapillary colloid osmotic gradient in hemodialysis patients, improvements in dialysis therapy," Contributions to Nephrology, vol. 74, pp. 170-175, 1989.

[31] J. Lindenfeld, N. M. Albert, J. P. Boehmer et al., "Executive summary: HFSA 2010 comprehensive heart failure practice guideline," Journal of Cardiac Failure, vol. 16, no. 6, pp. 475539, 2010.

[32] M. G. Felker, "Diuretic Optimization Strategies Evaluation (DOSE)," American College of Cardiology scientific session. Atlanta, 2010.

[33] G. S. Francis, R. M. Siegel, and S. R. Goldsmith, "Acute vasoconstrictor response to intravenous furosemide in patients with chronic congestive heart failure. Activation of the neurohumoral axis," Annals of Internal Medicine, vol. 103, no. 1, pp. 1-6, 1985.

[34] S. S. Gottlieb, D. C. Brater, I. Thomas et al., "BG9719 (CVT124), an A1 adenosine receptor antagonist, protects against the decline in renal function observed with diuretic therapy," Circulation, vol. 105, no. 11, pp. 1348-1353, 2002.

[35] K. V. Liang, A. W. Williams, E. L. Greene, and M. M. Redfield, "Acute decompensated heart failure and the cardiorenal syndrome," Critical Care Medicine, vol. 36, no. 1, pp. S75-S88, 2008.

[36] D. C. Brater, "Diuretic therapy," The New England Journal of Medicine, vol. 339, no. 6, pp. 387-395, 1998.

[37] P. Ginès and R. W. Schrier, "Renal failure in cirrhosis," The New England Journal of Medicine, vol. 361, no. 13, pp. 12791290, 2009.

[38] R. W. Schrier, A. Masoumi, and E. Elhassan, "Aldosterone: role in edematous disorders, hypertension, chronic renal failure, and metabolic syndrome," Clinical Journal of the American Society of Nephrology, vol. 5, no. 6, pp. 1132-1140, 2010.

[39] E. Braunwald, W. H. Plauth Jr., and A. G. Morrow, "A method for the detection and quantification of impaired sodium excretion. Results of an oral sodium tolerance test in normal subjects and in patients with heart disease," Circulation, vol. 32, pp. 223-231, 1965.

[40] J. Hensen, W. T. Abraham, J. A. Dürr, and R. W. Schrier, "Aldosterone in congestive heart failure: analysis of determinants and role in sodium retention," American Journal of Nephrology, vol. 11, no. 6, pp. 441-446, 1991.

[41] D. N. Juurlink, M. M. Mamdani, D. S. Lee et al., "Rates of hyperkalemia after publication of the randomized aldactone evaluation study," The New England Journal of Medicine, vol. 351, no. 6, pp. 543-551, 2004.

[42] B. Pitt, G. Bakris, L. M. Ruilope, L. DiCarlo, and R. Mukherjee, "Serum potassium and clinical outcomes in the eplerenone post-acute myocardial infarction heart failure efficacy and survival study (EPHESUS)," Circulation, vol. 118, no. 16, pp. 1643-1650, 2008.

[43] L. Wei, A. D. Struthers, T. Fahey, A. D. Watson, and T. M. Macdonald, "Spironolactone use and renal toxicity: population based longitudinal analysis," BMJ, vol. 340, p. c1768, 2010.

[44] S. R. Goldsmith, F. Brandimarte, and M. Gheorghiade, "Congestion as a therapeutic target in acute heart failure syndromes," Progress in Cardiovascular Diseases, vol. 52, no. 5, pp. 383-392, 2010.

[45] M. Gheorghiade, W. A. Gattis, C. M. O'Connor et al., "Effects of tolvaptan, a vasopressin antagonist, in patients hospitalized with worsening heart failure: a randomized controlled trial," Journal of the American Medical Association, vol. 291, no. 16, pp. 1963-1971, 2004.

[46] M. A. Konstam, M. Gheorghiade, J. C. Burnett Jr. et al., "Effects of oral tolvaptan in patients hospitalized for worsening heart failure: the EVEREST outcome trial," Journal of the American Medical Association, vol. 297, no. 12, pp. 1319-1331, 2007.

[47] S. R. Goldsmith, U. Elkayam, W. H. Haught, A. Barve, and W. $\mathrm{He}$, "Efficacy and safety of the vasopressin V1A/V2-receptor antagonist conivaptan in acute decompensated heart failure: a dose-ranging pilot study," Journal of Cardiac Failure, vol. 14, no. 8, pp. 641-647, 2008.

[48] Publication Committee for the VMAC Investigators (Vasodilatation in the Management of Acute $\mathrm{CHF}$ ), "Intravenous nesiritide vs nitroglycerin for treatment of decompensated 
congestive heart failure: a randomized controlled trial," Journal of the American Medical Association, vol. 288, no. 5, p. 577, 2002.

[49] J. D. Sackner-Bernstein, H. A. Skopicki, and K. D. Aaronson, "Risk of worsening renal function with nesiritide in patients with acutely decompensated heart failure," Circulation, vol. 111, no. 12, pp. 1487-1491, 2005.

[50] H. G. Riter, M. M. Redfield, J. C. Burnett, and H. H. Chen, "Nonhypotensive low-dose nesiritide has differential renal effects compared with standard-dose nesiritide in patients with acute decompensated heart failure and renal dysfunction," Journal of the American College of Cardiology, vol. 47, no. 11, pp. 2334-2335, 2006.

[51] G. Cotter, E. Metzkor, E. Kaluski et al., "Randomised trial of high-dose isosorbide dinitrate plus low-dose furosemide versus high-dose furosemide plus low-dose isosorbide dinitrate in severe pulmonary oedema," The Lancet, vol. 351, no. 9100, pp. 389-393, 1998.

[52] U. Elkayam, M. Janmohamed, M. Habib, and P. Hatamizadeh, "Vasodilators in the management of acute heart failure," Critical Care Medicine, vol. 36, no. 1, pp. S95-S105, 2008.

[53] G. Zemljic, M. Bunc, A. P. Yazdanbakhsh, and B. Vrtovec, "Levosimendan improves renal function in patients with advanced chronic heart failure awaiting cardiac transplantation," Journal of Cardiac Failure, vol. 13, no. 6, pp. 417-421, 2007.

[54] C. Liang, L. G. Sherman, and J. U. Doherty, "Sustained improvement of cardiac function in patients with congestive heart failure after short-term infusion of dobutamine," Circulation, vol. 69, no. 1, pp. 113-119, 1984.

[55] F. Dies, M. J. Krell, and P. Whitlow, "Intermittent dobutamine in ambulatory outpatients with chronic cardiac failure [Abstract]," Circulation, vol. 74, supplement II, p. 38, 1986.

[56] W. T. Abraham, K. F. Adams, G. C. Fonarow et al., "Inhospital mortality in patients with acute decompensated heart failure requiring intravenous vasoactive medications: an analysis from the Acute Decompensated Heart Failure National Registry (ADHERE)," Journal of the American College of Cardiology, vol. 46, no. 1, pp. 57-64, 2005.

[57] A. Ahmed and R. C. Campbell, "Epidemiology of chronic kidney disease in heart failure," Heart Failure Clinics, vol. 4, no. 4, pp. 387-399, 2008.

[58] J. W. Petersen and G. M. Felker, "Inotropes in the management of acute heart failure," Critical Care Medicine, vol. 36, no. 1, pp. S106-S111, 2008.

[59] M. Packer, "REVIVE II: multicenter placebo-controlled trial of levosimendan on clinical status in acutely decompensated heart failure," Circulation, vol. 112, pp. 3362-3364, 2005.

[60] B. M. Massie, C. M. O'Connor, M. Metra et al., "Rolofylline, an adenosine A1-receptor antagonist, in acute heart failure," The New England Journal of Medicine, vol. 363, no. 15, pp. 14191428, 2010.

[61] M. R. Costanzo, M. E. Guglin, M. T. Saltzberg et al., "Ultrafiltration versus intravenous diuretics for patients hospitalized for acute decompensated heart failure," Journal of the American College of Cardiology, vol. 49, no. 6, pp. 675-683, 2007.

[62] C. Giglioi, D. Landi, E Cecchi et al., "Effects of ULTRAfiltration vs. DIureticS on clinical, biohumoral and haemodynamic variables in patients with deCOmpensated heart failure: the ULTRADISCO study," European Journal of Heart Failure, vol. 13, no. 3, pp. 337-346, 2011. 


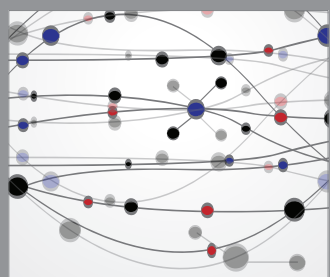

The Scientific World Journal
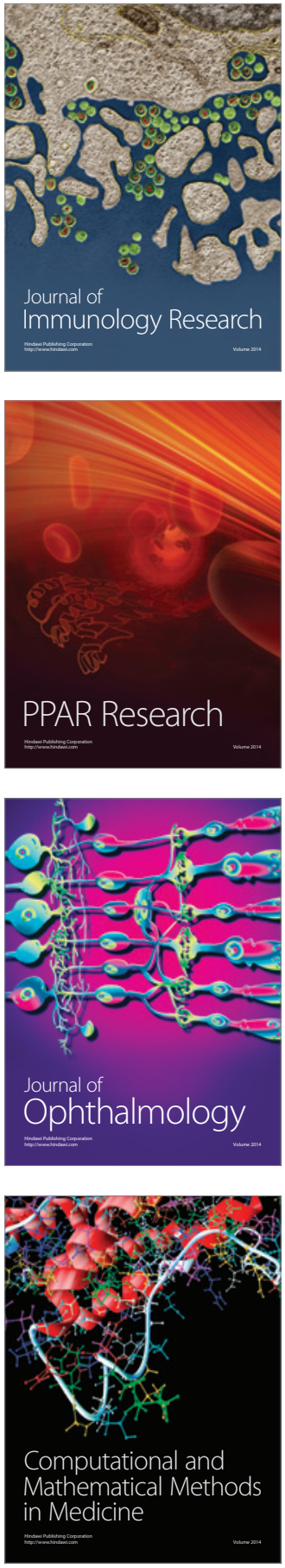

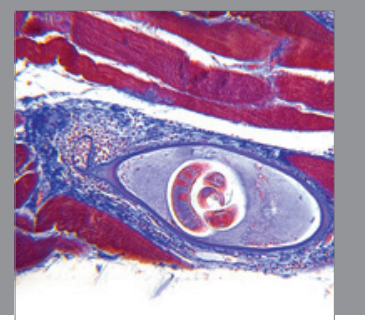

Gastroenterology

Research and Practice
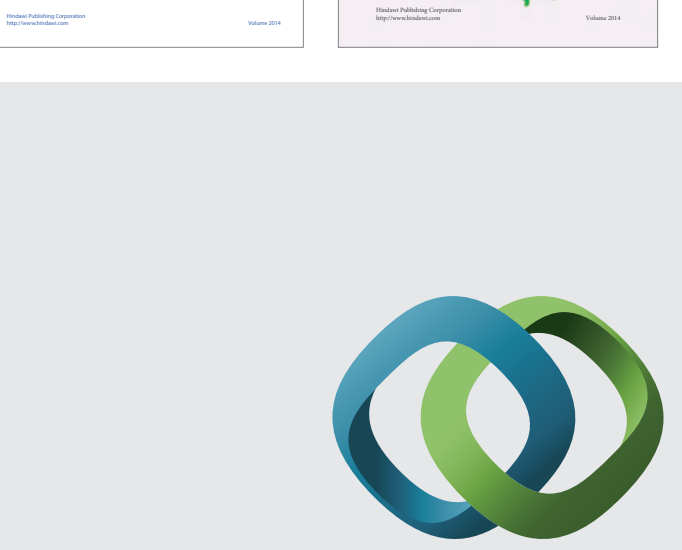

\section{Hindawi}

Submit your manuscripts at

http://www.hindawi.com
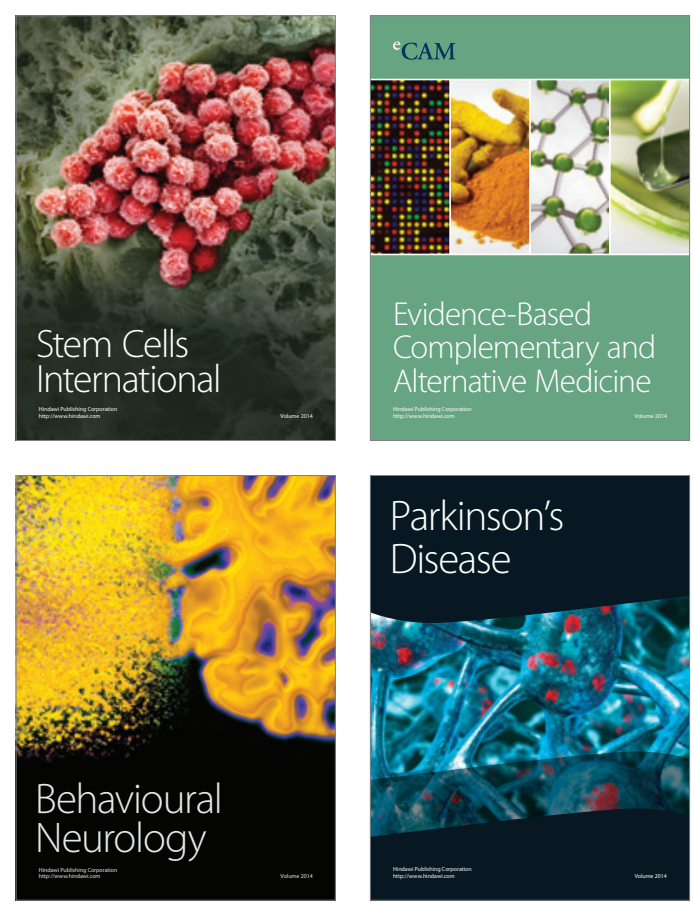

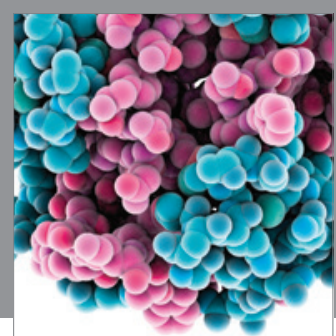

Journal of
Diabetes Research

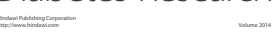

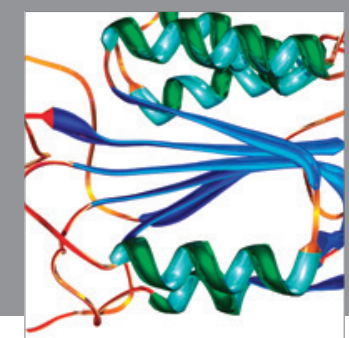

Disease Markers
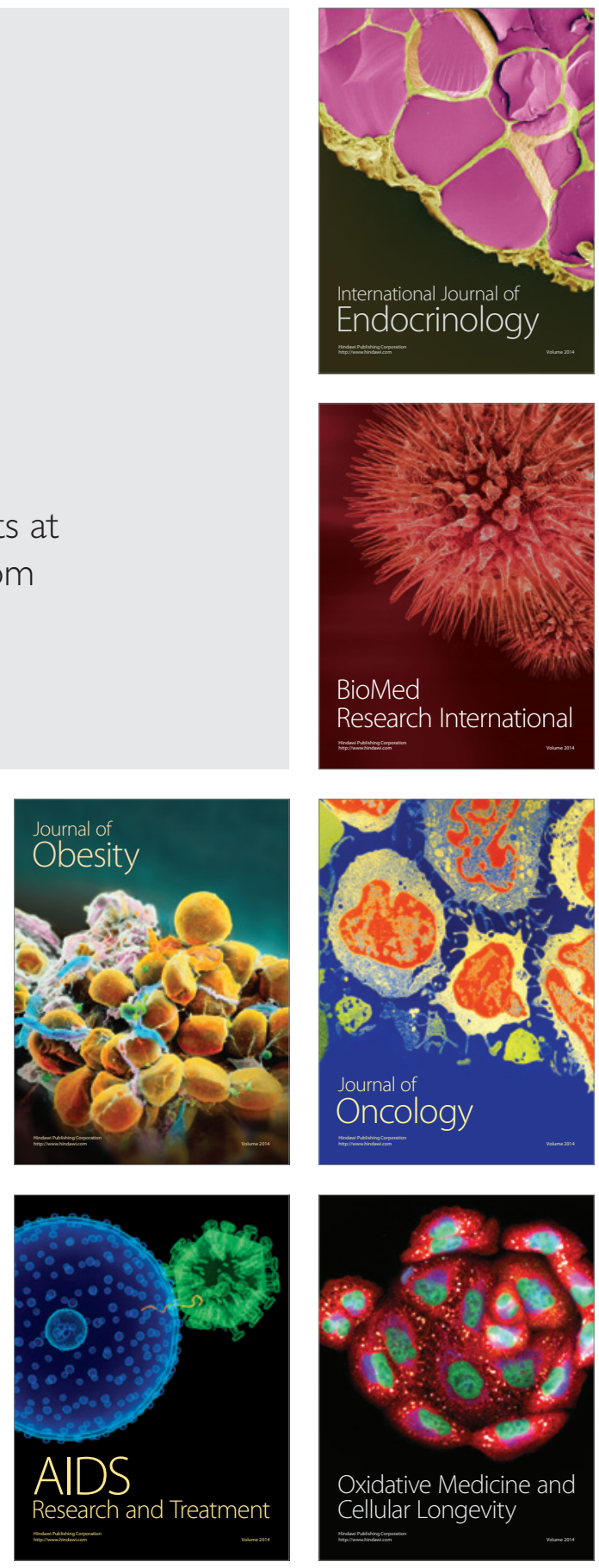